ESAIM: COCV 18 (2012) 877-888

DOI: $10.1051 / \mathrm{cocv} / 2011185$
ESAIM: Control, Optimisation and Calculus of Variations

www.esaim-cocv.org

\title{
STABILITY OF RETARDED SYSTEMS WITH SLOWLY VARYING COEFFICIENT
}

\author{
Michael IOSIF GIL ${ }^{1}$
}

\begin{abstract}
The "freezing" method for ordinary differential equations is extended to multivariable retarded systems with distributed delays and slowly varying coefficients. Explicit stability conditions are derived. The main tool of the paper is a combined usage of the generalized Bohl-Perron principle and norm estimates for the fundamental solutions of the considered equations.
\end{abstract}

Mathematics Subject Classification. 34K20

Received February 10, 2011. Revised July 8, 2011

Published online September 27, 2011.

\section{INTRODUCTION AND STATEMENT OF THE MAIN RESULT}

Stability of linear nonautonomous systems with delay has been investigated by many authors, $c f .[6,8]$, etc. Besides, the basic method for the stability analysis is the direct Lyapunov method. By that method many very strong results are obtained. But finding Lyapunov's type functionals for retarded systems is usually difficult. An interesting approach to obtaining explicit stability conditions for vector equations were established, in the papers $[13,14]$. That approach is based on deriving bounds for the norms of system solutions. About other explicit stability conditions see the fundamental survey [11]. But to the best of our knowledge, the general linear vector functional differential equations with slowly varying coefficients were almost not investigated in the available literature.

Let $\mathbb{R}^{n}$ be a real Euclidean space with the scalar product (.,.), Euclidean norm $\|\cdot\|_{n}=\sqrt{(., .)}$ and identity matrix $I$. For an $n \times n$-matrix $A,\|A\|_{n}=\sup _{z \in \mathbb{R}^{n}}\|A z\|_{n} /\|z\|_{n}$. Put $R_{+}=[0, \infty)$.

Consider in $\mathbb{R}^{n}$ the equation

$$
\dot{x}(t)=\int_{0}^{\eta} R(t, \mathrm{~d} \tau) x(t-\tau) \quad(t \geq 0 ; \dot{x}(t)=\mathrm{d} x / \mathrm{d} t)
$$

where $R(t, \delta)$ is an $n \times n$ - matrix-valued function defined on $R_{+} \times \Sigma$, where $\Sigma$ is the set of the Borel subsets of $[0, \eta]$. Besides, $R(.,$.$) is additive in the second argument and its variation in that argument is uniformly bounded$ with respect to the first argument on $R_{+}$. The integral in (1.1) is understood as the vector Riemann-Stieltjes integral, that is the limit of the sums

$$
\sum_{k=1}^{M-1} R\left(t, \Delta_{k}^{(M)}\right) x\left(t-\tau_{k}^{(M)}\right) \text { as } \max _{k}\left|\Delta_{k}^{(M)}\right| \rightarrow 0 .
$$

Keywords and phrases. Linear retarded systems, stability, generalized Bohl-Perron principle

1 Department of Mathematics, Ben Gurion University of the Negev, P.O. Box 653, 84105 Beer-Sheva, Israel. gilmi@bezeqint.net 
Here $\Delta_{k}^{(M)}=s_{k+1}^{(M)}-s_{k}^{(M)}, 0=s_{0}^{(M)}<s_{1}^{(M)}<\ldots<s_{M}^{(M)}=\eta$ and $\tau_{k}^{(M)} \in \Delta_{k}^{(M)}(k=1, \ldots, M)$.

We extend the "freezing" method for ordinary differential equations $c f .[1,7,12]$ and [3], Section 3.2, to equation (1.1). Besides, explicit exponential stability conditions are derived. They considerably generalize and improve Theorem 9.3.1 from [3], and generalize Theorem 4.15 from [5].

It is assumed that there is a positive constant $q$, such that

$$
\int_{0}^{\eta}\|R(t, \mathrm{~d} \tau)-R(s, \mathrm{~d} \tau)\|_{n} \leq q|t-s|(t, s \geq 0) .
$$

The integral in (1.2) means the limit of the sums

$$
\sum_{k=1}^{M-1}\left\|R\left(t, \Delta_{k}^{(M)}\right)-R\left(s, \Delta_{k}^{(M)}\right)\right\|_{n}
$$

as $\max _{k}\left|\Delta_{k}^{(M)}\right| \rightarrow 0$. For example, consider the equation

$$
\dot{x}(t)=\sum_{k=1}^{m} A_{k}(t) \int_{0}^{\eta} x(t-\tau) \mathrm{d} \mu_{k}(\tau)
$$

where $\mu_{k}$ are nondecreasing scalar functions and $A_{k}(t)$ are variable $n \times n$-matrices with the properties

$$
\left\|A_{k}(t)-A_{k}(s)\right\|_{n} \leq q_{k}|t-s|(t, s \geq 0) \text { and } \sup _{t \geq 0}\left\|A_{k}(t)\right\|_{n}<\infty
$$

with $q_{k}=$ const. $\geq 0(k=1, \ldots, m)$. Then $(1.2)$ holds, with

$$
q=\sum_{k=1}^{m} q_{k} \operatorname{var}\left(\mu_{k}\right),
$$

where $\operatorname{var}\left(\mu_{k}\right)$ denotes the variation of $\mu_{k}$.

A solution of (1.1) is an absolutely continuous function which satisfies that equation on $(0, \infty)$ almost everywhere with the initial condition

$$
x(t)=\phi(t) \quad(-\eta \leq t \leq 0)
$$

for a given continuous vector function $\phi(t)$ defined on $[-\eta, 0]$. Existence results and stability definitions can be found for instance in $[6,8]$.

Recall that a differentiable in $t$ function $G\left(t, t_{1}\right) \quad\left(t \geq t_{1} \geq 0\right)$ is the fundamental solution to (1.1) if it satisfies that equation in $t$ and the initial conditions

$$
G(t, t)=I, G\left(t, t_{1}\right)=0\left(t<t_{1}, t_{1} \geq 0\right) .
$$

For a fixed $s \geq 0$, consider the "frozen" equation

$$
\dot{y}(t)=\int_{0}^{\eta} R(s, \mathrm{~d} \tau) y(t-\tau)(t>0) .
$$

Since (1.6) is an autonomous equation, the fundamental solution $\hat{G}_{s}\left(t, t_{1}\right)$ to equation (1.6) is defined by $\hat{G}_{s}\left(t, t_{1}\right)=\hat{G}_{s}\left(t-t_{1}\right)$, where $\hat{G}_{s}(t)$ is a matrix-valued function satisfying that equation and the initial conditions

$$
\hat{G}_{s}(0+)=I, \hat{G}_{s}(t)=0(t<0) .
$$

Now we are in a position to formulate our main result. 
Theorem 1.1. Let the conditions (1.2) and

$$
\chi:=\sup _{s \geq 0} \int_{0}^{\infty} t\left\|\hat{G}_{s}(t)\right\|_{n} \mathrm{~d} t<\frac{1}{q}
$$

hold. Then equation (1.1) is exponentially stable.

This theorem is proved in Section 5.

\section{EquATIONS "CLOSE" TO ORDINARY DIFFERENTIAL ONES}

Let us point a stability criterion which is more convenient than Theorem 1.1 in the case of the equation

$$
\dot{x}(t)=A(t) x(t)+\int_{0}^{\eta} R_{0}(t, \mathrm{~d} \tau) x(t-\tau)(t>0),
$$

where $R_{0}(t, \delta)$ is an $n \times n$ matrix-valued function defined on $R_{+} \times \Sigma$ and $A(t)$ for any $t \geq 0$ is an $n \times n$ Hurwitzian matrix. Besides, $R_{0}(t,$.$) is additive in the second argument and its variation is uniformly bounded$ in $t \in[0, \infty)$. That is,

$$
V\left(R_{0}\right):=\sup _{s \geq 0} \int_{0}^{\eta}\left\|R_{0}(s, \mathrm{~d} \tau)\right\|_{n}<\infty
$$

It is assumed that

$$
\|A(t)-A(s)\|_{n} \leq q_{0}|t-s|(t, s \geq 0) .
$$

Theorem 2.1. Let the conditions (2.2),

$$
\nu_{A}:=\sup _{s \geq 0} \int_{0}^{\infty}\left\|\mathrm{e}^{A(s) t}\right\|_{n} \mathrm{~d} t<\frac{1}{V\left(R_{0}\right)}
$$

and

$$
\hat{\chi}_{0}:=\sup _{s \geq 0} \int_{0}^{\infty} t\left\|\mathrm{e}^{A(s) t}\right\|_{n} \mathrm{~d} t<\frac{1-\nu_{A} V\left(R_{0}\right)}{q_{0}}
$$

hold. Then equation (2.1) is exponentially stable.

This theorem is also proved in Section 5. For instance, consider the equation

$$
\dot{x}(t)=A(t) x(t)+\sum_{k=1}^{m} A_{k}(t) x\left(t-h_{k}\right) \quad(t \geq 0)
$$

where $h_{k}=$ const $>0$ and $A_{k}(t)$ are $n \times n$-matrices with the properties $\sup _{t \geq 0}\left\|A_{k}(t)\right\|_{n}<\infty(k=1, \ldots, m)$. In the considered case

$$
V\left(R_{0}\right)=\sup _{s} \sum_{k=1}^{m}\left\|A_{k}(s)\right\|_{n} .
$$

About simple estimates for $\left\|\mathrm{e}^{A(s) t}\right\|_{n}$ see [3], Section 3.2. 


\section{Equation With one Distributed Delay}

In this section we illustrate Theorem 1.1 in the case of the system

$$
\dot{x}(t)=A(t) \int_{0}^{\eta} x(t-\tau) \mathrm{d} \mu(\tau),
$$

where $\mu$ is a scalar nondecreasing function of a bounded variation $\operatorname{var}(\mu)$, and $A(t)$ is a negative definite Hermitian $n \times n$-matrix, satisfying (2.2). Hence, condition (1.2) holds with

$$
q=q_{0} \operatorname{var}(\mu) .
$$

Furthermore, for a fixed $s \geq 0$, consider the equation

$$
\dot{x}(t)=A(s) \int_{0}^{\eta} x(t-\tau) \mathrm{d} \mu(\tau) .
$$

Put

$$
E_{\mu} w(t)=\int_{0}^{\eta} w(t-\tau) \mathrm{d} \mu(\tau) .
$$

Reduce equation (3.3) to the diagonal form:

$$
\dot{x}_{j}(t)=\lambda_{j}(s) E_{\mu} x_{j}(t) \quad(j=1, \ldots, n),
$$

where $\lambda_{j}(s)$ are the eigenvalues of $A(s)$. Let $X_{s, j}(t)$ be the fundamental solution of the scalar equation (3.4). Assume that

$$
J_{j}(s):=\int_{0}^{\infty}\left|X_{s, j}(t)\right| \mathrm{d} t<\infty \quad(j=1, \ldots, n) .
$$

Then the fundamental solution $\hat{G}_{s}(t)$ to (3.3) satisfies the equality $\int_{0}^{\infty}\left\|\hat{G}_{s}(t)\right\|_{n} \mathrm{~d} t=\max _{j} J_{j}(s)$. The inequality

$$
\chi \leq\left(1+v_{1}(\mu)\right) \sup _{s \geq 0, j=1, \ldots, n} J_{j}^{2}(s) \text { where } v_{1}(\mu):=\int_{0}^{\eta} \tau \mathrm{d} \mu(\tau),
$$

holds according to Lemma 5.8 proved below. Now Theorem 1.1 implies

Theorem 3.1. If the conditions (2.2), (3.5) and

$$
\operatorname{var}(\mu) q_{0}\left(1+v_{1}(\mu)\right) \sup _{s \geq 0, j=1, \ldots, n} J_{j}^{2}(s)<1
$$

hold, then system (3.1) is exponentially stable.

Furthermore, let

$$
k(z)=z+\int_{0}^{\eta} \exp (-z \tau) \mathrm{d} \mu(\tau)(z \in \mathbb{C}) .
$$

So $k(z)$ is the characteristic function of the scalar equation

$$
\dot{y}+\int_{0}^{\eta} y(t-\tau) \mathrm{d} \mu(\tau)=0 .
$$

The fundamental solution of (3.6) is

$$
X_{\mu}(t)=\frac{1}{2 \pi i} \int_{c-i \infty}^{c+i \infty} \mathrm{e}^{z t} k^{-1}(z) \mathrm{d} z \quad(c=\text { const. }) .
$$


Hence,

$$
\frac{1}{k(z)}=\int_{0}^{\infty} \mathrm{e}^{-z t} X_{\mu}(t) \mathrm{d} t
$$

If

$$
\text { eq } \operatorname{var}(\mu)<1,
$$

then as it was to shown in the paper [4], we have $X_{\mu}(t) \geq 0$ and $X_{\mu}(t) \rightarrow 0$ as $t \rightarrow \infty$. So

$$
\frac{1}{|k(i \omega)|} \leq \int_{0}^{\infty} X_{\mu}(t) \mathrm{d} t=\frac{1}{k(0)}=\frac{1}{\operatorname{var}(\mu)}(\omega \in \mathbb{R}) .
$$

Assume that

$$
\sup _{s \geq 0, k=1, \ldots, n}\left|\lambda_{k}(s)\right|<\frac{1}{e \operatorname{var}(\mu) \eta}
$$

and

$$
\zeta:=\min _{k=1, \ldots, n \geq 0} \inf _{s \geq 0}\left|\lambda_{k}(s)\right|>0 .
$$

Then by (3.8) we have $J_{j}(s) \leq \frac{1}{\operatorname{var}(\mu) \zeta}(j \leq n)$. Now Theorem 3.1 implies

Corollary 3.2. Let the conditions (2.2), (3.9) and

$$
q_{0}\left(1+v_{1}(\mu)\right)<\operatorname{var}(\mu) \zeta^{2}
$$

hold. Then (3.1) is exponentially stable.

\section{EXAMPLE}

Consider the system

$$
\begin{aligned}
& \dot{x}_{1}+\left(a_{1}+b_{1} \cos (\omega t)\right) x_{1}(t-\eta)+c \sin (\omega t) x_{2}(t-\eta)=0, \\
& \dot{x}_{2}+\left(a_{2}+b_{2} \cos (\omega t)\right) x_{2}(t-\eta)+c \sin (\omega t) x_{1}(t-\eta)=0,
\end{aligned}
$$

with positive constants $\omega, a_{1}, a_{2}, b_{1}, b_{2}$ and $c$, satisfying the condition $b_{k}+c<a_{k}(k=1,2)$. Rewrite (4.1) as $\dot{x}=A_{2}(t) x(t-\eta)$, where

$$
A_{2}(t)=-\left(\begin{array}{cc}
a_{1}+b_{1} \cos (\omega t) & c \sin (\omega t) \\
c \sin (\omega t) & a_{2}+b_{2} \cos (\omega t)
\end{array}\right) .
$$

Simple calculations show that this matrix satisfies (2.2) with

$$
q_{0} \leq q_{\omega}=\omega\left[b_{1}^{2}+b_{2}^{2}+2 c^{2}\right]^{1 / 2} .
$$

By the Herschgorin theorem, $c f$. [10], the eigenvalues $\lambda_{1,2}(t)$ of $A_{2}(t)$ satisfy the inequalities $\left|a_{k}+\lambda_{k}(t)\right| \leq b_{k}+c$, and therefore

$$
\left|\lambda_{k}(t)\right| \leq \max _{k}\left(a_{k}+b_{k}+c\right), \quad \text { and }\left|\lambda_{k}(t)\right| \geq \zeta_{0}:=\min _{k}\left(a_{k}-b_{k}-c\right)(k=1,2) .
$$

Thus $\zeta \geq \zeta_{0}$. In addition, $\operatorname{var}(\mu)=1$ and $v_{1}(\mu)=\eta$. So $(3.9)$ is provided by

$$
c+\max _{k}\left(a_{k}+b_{k}\right)<\frac{1}{e \eta}
$$

and (3.10) is provided by

$$
q_{\omega}(1+\eta)<\zeta_{0}^{2}
$$


Consequently, if (4.2) and (4.3) hold, then by Corollary 3.2 system (4.1) is exponentially stable.

For instance, consider the system

$$
\begin{aligned}
& \dot{x}_{1}+(10+\cos (\omega t)) x_{1}(t-0.02)+\sin (\omega t) x_{2}(t-0.02)=0 \\
& \dot{x}_{2}+(10+\cos (\omega t)) x_{2}(t-0.02)+\sin (\omega t) x_{1}(t-0.02)=0 .
\end{aligned}
$$

So

$$
a_{1}=a_{2}=10, b_{1}=b_{2}=c_{1}=c_{2}=1, \eta=0.02 \text { and } q_{0} \leq 2 \omega .
$$

By the above mentioned Herschgorin theorem, the eigenvalues $\lambda_{1,2}(t)$ satisfy the inequalities $\left|10+\lambda_{k}(t)\right| \leq 2$, and therefore

$$
\left|\lambda_{k}(t)\right| \leq 12 \text {, and }\left|\lambda_{k}(t)\right| \geq \zeta_{0}=8(k=1,2) .
$$

In addition, $\operatorname{var}(\mu)=1$ and $v_{1}(\mu)=0.02$. So (4.2) holds, since $12<1 / 0.02 e$. Thus condition (4.3) holds, if $2.04 \omega<64$. Consequently, system (4.4) is exponentially stable, provided

$$
\omega<\frac{64}{2.04}
$$

\section{ProOFS}

Let $C(\Omega)=C\left(\Omega, \mathbb{R}^{n}\right)$ be the space of continuous real vector functions $f(t)=\left(f_{j}(t)\right)_{j=1}^{n}$ defined and bounded on a set $\Omega$ with the sup-norm

$$
\|f\|_{C(\Omega)}=\sup _{t \in \Omega}\|f(t)\|_{n} .
$$

By $\hat{C}(\Omega)=\hat{C}\left(\Omega, \mathbb{R}^{n}\right)$ we denote the same space with the norm

$$
\|f\|_{\hat{C}(\Omega)}=\max _{j=1, \ldots, n} \sup _{t \in \Omega}\left|f_{j}(t)\right| .
$$

Introduce in $\hat{C}(\Omega)$ the operator

$$
A f(t)=\int_{\Omega} K(t, s) f(s) \mathrm{d} s,
$$

where $K(t, s)=\left(K_{j k}(t, s)\right)_{j, k=1}^{n}$ is a real matrix function defined on $\Omega \times \Omega$, continuous in the first argument and satisfying

$$
\tau_{A}:=\max _{j=1, \ldots, n} \sup _{t \in \Omega} \int_{\Omega} \sum_{k=1}^{n}\left|K_{j k}(t, s)\right| \mathrm{d} s<\infty .
$$

Put $\|A\|_{\hat{C}(\Omega)}=\sup _{w \in C(\Omega)}\|A w\|_{\hat{C}(\Omega)} /\|w\|_{\hat{C}(\Omega)}$.

Lemma 5.1. Let $A$ be defined by (5.1) and condition (5.2) hold. Then $\|A\|_{\hat{C}(\Omega)}=\tau_{A}$.

Proof. It is simple to show that

$$
\|A\|_{\hat{C}(\Omega)} \leq \max _{t, j} \int_{\Omega} \sum_{k=1}^{n}\left|K_{j k}(t, s)\right| \mathrm{d} s=\tau_{A} .
$$

Let $j_{0}$ and $t_{0} \in \Omega$ be such that

$$
\tau_{A}=\int_{\Omega} \sum_{k=1}^{n}\left|K_{j_{0} k}\left(t_{0}, s\right)\right| \mathrm{d} s .
$$


Take $h(s)=\left(h_{k}(s)\right)_{k=1}^{n}$ with $h_{k}(s)=$ signum $K_{j_{0} k}\left(t_{0}, s\right)$. Then we have

$$
(A h)_{j}(t):=\int_{\Omega} \sum_{k=1}^{n} K_{j k}(t, s) h_{k}(s) \mathrm{d} s=\int_{\Omega} \sum_{k=1}^{n} K_{j k}(t, s)\left(\operatorname{signum} K_{j_{0} k}\left(t_{0}, s\right)\right) \mathrm{d} s
$$

provided $j \neq j_{0}$. In addition,

$$
(A h)_{j_{0}}\left(t_{0}\right)=\int_{\Omega} \sum_{k=1}^{n}\left|K_{j_{0} k}\left(t_{0}, s\right)\right| \mathrm{d} s
$$

But according to (5.2),

$$
\left|(A h)_{j}(t)\right| \leq \int_{\Omega} \sum_{k=1}^{n}\left|K_{j_{0} k}\left(t_{0}, s\right)\right| \mathrm{d} s \quad\left(j \neq j_{0}\right) .
$$

So $\|A h\|_{\hat{C}(\Omega)}=\tau_{A}$. Taking into account that $\left|\operatorname{signum} K_{j_{0} k}\left(t_{0}, s\right)\right|=1$, we have $\|h\|_{\hat{C}(\Omega)}=1$ and therefore $\|A h\|_{\hat{C}(\Omega)} /\|h\|_{\hat{C}(\Omega)}=\tau_{A}$. This and (5.3) prove the result.

Furthermore, due to the variation of constants formula the equation

$$
\dot{y}(t)=\int_{0}^{\eta} R(t, \mathrm{~d} \tau) y(t-\tau)+f(t) \quad(t \geq 0)
$$

with a given vector function $f$ and the zero initial condition

$$
y(t)=0(t \leq 0)
$$

is equivalent to the equation

$$
y(t)=\int_{0}^{t} G(t, \tau) f(\tau) \mathrm{d} \tau
$$

Let $G_{j k}(t, s)$ be the entries of $G(t, s)$.

Corollary 5.2. Let a solution of problem (5.5), (5.6) be bounded on $R_{+}$for any $f \in C\left(R_{+}\right)$. Then

$$
\max _{t \geq 0, j=1, \ldots, n} \int_{0}^{t} \sum_{k=1}^{n}\left|G_{j k}(t, s)\right| \mathrm{d} s<\infty .
$$

Indeed, this result at once follows from the previous lemma.

Let $x(t)$ be a solution of problem (1.1), (1.5). Put

$$
\theta(t)= \begin{cases}\phi(0) & \text { if } t \geq 0 \\ \phi(t) & \text { if }-\eta \leq t \leq 0\end{cases}
$$

and $y_{0}(t)=x(t)-\theta(t)$. We can write $d \theta(t) / \mathrm{d} t=0 \quad(t \geq 0)$ and

$$
\dot{y}_{0}(t)=\int_{0}^{\eta} R(t, \mathrm{~d} \tau) y_{0}(t-\tau)+\psi(t) \quad(t \geq 0),
$$

where

$$
\psi(t)=\int_{0}^{\eta} R(t, \mathrm{~d} \tau) \theta(t-\tau) .
$$

Hence, by the variation of constants formula we have

$$
y_{0}(t)=\int_{0}^{t} G(t, s) \psi(s) \mathrm{d} s \quad(t \geq 0) .
$$

But $x(t)=y_{0}(t)+\theta(t)$. We thus have proved the following result. 
Lemma 5.3. A solution of problem (1.1), (1.5) can be represented by the equality

$$
x(t)=\phi(0)+\int_{0}^{t} G\left(t, t_{1}\right) \psi\left(t_{1}\right) \mathrm{d} t_{1} \quad(t \geq 0) .
$$

Following [5], page 371, we will say that equation (5.5) satisfies the Perron condition, if for any $f \in C\left(R_{+}\right)$, a solution of problem (5.5), (5.6) is bounded.

Lemma 5.4. If equation (5.5) satisfies the Perron condition, then a solution of (1.1) is bounded.

Proof. We have

$$
\begin{aligned}
\left\|\int_{0}^{t} G(t, s) \psi(s) \mathrm{d} s\right\|_{n} & \leq\left\|\int_{0}^{t} G(t, s) \int_{0}^{\eta} R(s, \mathrm{~d} \tau) \hat{\phi}(s-\tau) \mathrm{d} s\right\|_{n} \leq\|\phi\|_{C[-\eta, 0]} \int_{0}^{t}\|G(t, s)\|_{n} \int_{0}^{\eta}\|R(s, \mathrm{~d} \tau)\|_{n} \mathrm{~d} s \\
& \leq V(R)\|\phi\|_{C[-\eta, 0]} \sup _{t \geq 0} \int_{0}^{t}\|G(t, s)\|_{n} \mathrm{~d} s,
\end{aligned}
$$

where

$$
V(R):=\sup _{t \geq 0} \int_{0}^{\eta}\|R(t, \mathrm{~d} \tau)\|_{n} \mathrm{~d} s .
$$

So by Corollary 5.2 and the previous lemma we have the inequality

$$
\|x\|_{C\left(R_{+}\right)} \leq m_{0}\|\phi\|_{C[-\eta, 0]},
$$

where

$$
m_{0}:=1+V(R) \sup _{t \geq 0} \int_{0}^{t}\|G(t, s)\|_{n} \mathrm{~d} s
$$

This proves the lemma.

Lemma 5.5. If equation (5.5) satisfies the Perron condition, then (1.1) is exponentially stable.

Proof. Substituting

$$
x_{1}(t)=\mathrm{e}^{\epsilon t} x(t)
$$

with an $\epsilon>0$ into (1.1), we obtain the equation

$$
\dot{x}_{1}(t)=\epsilon x_{1}(t)+\int_{0}^{\eta} \mathrm{e}^{\epsilon \tau} R(t, \mathrm{~d} \tau) x_{1}(t-\tau) \quad(t>0) .
$$

Put

$$
\hat{G} f(t)=\int_{0}^{t} G(t, s) f(s) \mathrm{d} s .
$$

Under the hypothesis of the theorem, $\hat{G}$ is defined on the whole space $C(0, \infty)$. By the Banach theorem [9], Section $2, \hat{G}$ is bounded on $C(0, \infty)$. Clearly, it is bounded on $C(0, T), T<\infty$. Consider also the equation

$$
\dot{y}_{\epsilon}(t)=\epsilon y_{\epsilon}(t)+\int_{0}^{\eta} \mathrm{e}^{\epsilon \tau} d_{\tau} R(t, \mathrm{~d} \tau) y_{\epsilon}(t-\tau)+f(t) .
$$

According to (5.5),

$$
\begin{aligned}
\frac{\mathrm{d}}{\mathrm{d} t}\left(y-y_{\epsilon}\right)(t) & =\int_{0}^{\eta} d_{\tau} R(t, \mathrm{~d} \tau) y(t-\tau)-\epsilon y_{\epsilon}(t)-\int_{0}^{\eta} \mathrm{e}^{\epsilon \tau} d_{\tau} R(t, \mathrm{~d} \tau) y_{\epsilon}(t-\tau) \\
& =\int_{0}^{\eta} d_{\tau} R(t, \mathrm{~d} \tau)\left(y(t-\tau)-y_{\epsilon}(t)\right)=f_{\epsilon}(t),
\end{aligned}
$$


where

$$
f_{\epsilon}(t)=-\epsilon y_{\epsilon}(t)+\int_{0}^{\eta}\left(1-\mathrm{e}^{\epsilon \tau}\right) d_{\tau} R(t, \mathrm{~d} \tau) y_{\epsilon}(t-\tau)
$$

Consequently, $y-y_{\epsilon}=\hat{G} f_{\epsilon}$. For simplicity put $|y|_{T}=\sup _{0 \leq t \leq T}\|y(t)\|_{n}(T<\infty)$. Then

$$
\left|f_{\epsilon}\right|_{T} \leq\left|y_{\epsilon}\right|_{T}\left(\epsilon+V(R)\left(\mathrm{e}^{\epsilon \eta}-1\right)\right) .
$$

So

$$
\left|y_{\epsilon}\right|_{T} \leq|y|_{T}+\left|y_{\epsilon}\right|_{T}|\hat{G}|_{T}\left(\epsilon+V(R)\left(\mathrm{e}^{\epsilon \eta}-1\right)\right) .
$$

Thus for a sufficiently small $\epsilon$,

$$
\left|y_{\epsilon}\right|_{T} \leq|y|_{T}\left(1-|\hat{G}|_{T}\left(\epsilon+V(R)\left(\mathrm{e}^{\epsilon \eta}-1\right)\right)\right)^{-1} .
$$

Hence, letting $t \rightarrow \infty$, by the Banach-Steinhaus theorem, we get

$$
\left\|y_{\epsilon}\right\|_{C(0, \infty)} \leq\|y\|_{C(0, \infty)}\left(1-\|\hat{G}\|_{C(0, \infty)}\left(\epsilon+V(R)\left(\mathrm{e}^{\epsilon \eta}-1\right)\right)\right)^{-1} .
$$

By the previous lemma, $x_{1}$ is bounded. Now (5.10) proves the exponential stability. As claimed.

The latter lemma generalizes Theorem 4.15 from [5], and therefore it generalizes the Bohl-Perron principle for ordinary differential equations to functional differential equations.

Proof of Theorem 1.1. For a vector-valued function $u$ defined on $[-\eta, \infty)$ and a fixed $s \geq 0$, put

$$
E(s) u(t)=\int_{0}^{\eta} R(s, \mathrm{~d} \tau) u(t-\tau)
$$

Then (5.5) can be written as

$$
\dot{y}(t)=E(s) y(t)+[E(t)-E(s)] y(t)+f(t) .
$$

By the Variation of Constants formula

$$
y(t)=\int_{0}^{t} \hat{G}_{s}\left(t-t_{1}\right)\left[\left(E\left(t_{1}\right)-E(s)\right) y\left(t_{1}\right)+f\left(t_{1}\right)\right] \mathrm{d} t_{1} .
$$

Thanks to condition (1.2), for all $s \leq t$ we obtain

$$
\|[E(t)-E(s)] y(t)\|_{n}=\left\|\int_{0}^{\eta}[R(t, \mathrm{~d} \tau)-R(s, \mathrm{~d} \tau)] y(t-\tau)\right\|_{n} \leq q|t-s|\|y\|_{C(0, t)} .
$$

Note that for an $\epsilon>0$, we have

$$
\int_{0}^{\infty}\left\|\hat{G}_{s}(t)\right\|_{n} \mathrm{~d} t \leq \int_{0}^{\epsilon}\left\|\hat{G}_{s}(t)\right\|_{n} \mathrm{~d} t+\frac{1}{\epsilon} \int_{\epsilon}^{\infty} t\left\|\hat{G}_{s}(t)\right\|_{n} \leq c_{1}
$$

where

$$
c_{1}=\sup _{s \geq 0} \int_{0}^{\epsilon}\left\|\hat{G}_{s}(t)\right\|_{n} \mathrm{~d} t+\frac{1}{\epsilon} \chi .
$$

Now (5.12) and (5.13) imply

$$
\|y\|_{C(0, t)} \leq c_{1}\|f\|_{C\left(R_{+}\right)}+\|y\|_{C(0, t)} \int_{0}^{t}\left\|\hat{G}_{s}\left(t-t_{1}\right)\right\|_{n} q\left|t_{1}-s\right| \mathrm{d} t_{1} .
$$


Take $s=t$. Then

$$
\begin{aligned}
\int_{0}^{t}\left\|\hat{G}_{s}\left(t-t_{1}\right)\right\|_{n}\left|t_{1}-s\right| \mathrm{d} t_{1} & =\int_{0}^{t}\left\|\hat{G}_{t}\left(t-t_{1}\right)\right\|_{n}\left(t-t_{1}\right) \mathrm{d} t_{1} \\
& =\int_{0}^{t}\left\|\hat{G}_{t}(u)\right\|_{n} u \mathrm{~d} u \leq \int_{0}^{\infty}\left\|\hat{G}_{t}(u)\right\|_{n} u \mathrm{~d} u \leq \chi .
\end{aligned}
$$

Thus $\|y\|_{C(0, t)} \leq c_{1}\|f\|_{C\left(R_{+}\right)}+q \chi\|y\|_{C(0, t)}$. Now the condition $q \chi<1$ implies

$$
\|y\|_{C\left(R_{+}\right)} \leq \frac{c_{1}\|f\|_{C\left(R_{+}\right)}}{1-q \chi}
$$

So for any bounded $f$ the solution to (5.5), (5.6) is uniformly bounded. Now Lemma 5.5 proves the theorem.

To prove Theorem 2.1, consider the equation

$$
\dot{x}(t)=A(t) x(t)+\int_{0}^{\eta} d_{\tau} R_{0}(t, \tau) x(t-\tau)+f(t)
$$

and denote by $U(t, s)(t \geq s \geq 0)$ the evolution operator of the equation

$$
\dot{y}(t)=A(t) y(t) .
$$

Introduce the operator $\hat{U}$ by

$$
\hat{U} f(t)=\int_{0}^{t} U(t, s) f(s) \mathrm{d} s .
$$

Lemma 5.6. Let the condition

$$
\|\hat{U}\|_{C(0, \infty)}<\frac{1}{V\left(R_{0}\right)}
$$

hold. Then any solution of (5.14) with $f \in C\left(R_{+}\right)$and the zero initial condition satisfies the inequality

$$
\|x\|_{C(0, \infty)} \leq\left(1-V\left(R_{0}\right)\|\hat{U}\|_{C(0, \infty)}\right)^{-1}\|\hat{U}\|_{C(0, \infty)}\|f\|_{C(0, \infty)} .
$$

Proof. Put

$$
E_{0} x(t)=\int_{0}^{\eta} d_{\tau} R_{0}(t, \tau) x(t-\tau)
$$

Equation (5.14) is equivalent to the following one:

$$
x(t)=\int_{0}^{t} U(t, s)\left(E_{0} x(s)+f(s)\right) \mathrm{d} s .
$$

Hence,

$$
\|x\|_{C(0, \infty)} \leq\|\hat{U}\|_{C(0, \infty)}\left(\left\|E_{0} x\right\|_{C(0, \infty)}+\|f\|_{C(0, \infty)}\right) .
$$

It is simple to check that $\left\|E_{0} x\right\|_{C(0, \infty)} \leq V\left(R_{0}\right)\left\|E_{0} x\right\|_{C(0, \infty)}$. Hence, by (5.16) we arrive at the required result.

The previous lemma and Lemma 5.5 imply

Corollary 5.7. Let conditions (2.2) and (5.16) hold. Then equation (2.1) is exponentially stable. 
Proof of Theorem 2.1. Consider the equation

$$
\dot{x}(t)=A(t) x(t)+f(t)
$$

with the zero inintial condition $x(0)=0$. Rewrite it as

$$
\dot{x}(t)=A(s) x(t)+(A(t)-A(s)) x(t)+f(t) .
$$

Hence

$$
x(t)=\int_{0}^{t} \mathrm{e}^{A(s)\left(t-t_{1}\right)}\left[\left(A\left(t_{1}\right)-A(s)\right) x\left(t_{1}\right)+f\left(t_{1}\right)\right] \mathrm{d} t_{1} .
$$

Take $s=t$. Then

$$
\|x(t)\|_{n} \leq \int_{0}^{t}\left\|\mathrm{e}^{A(t)\left(t-t_{1}\right)}\right\|\left\|\left(A\left(t_{1}\right)-A(t)\right) x\left(t_{1}\right)\right\| \mathrm{d} t_{1}+c_{0},
$$

where

$$
c_{0}:=\sup _{s} \sup _{t} \int_{0}^{t}\left\|\mathrm{e}^{A(s)\left(t-t_{1}\right)}\right\|_{n}\left\|f\left(t_{1}\right)\right\|_{n} \mathrm{~d} t_{1} \leq\|f\|_{C\left(R_{+}\right)} \sup _{s} \int_{0}^{\infty}\left\|\mathrm{e}^{A(s) t_{1}}\right\|_{n} \mathrm{~d} t_{1} \leq \nu_{A}\|f\|_{C\left(R_{+}\right)} .
$$

Thus, by (2.2), for any $T<\infty$,

$$
\begin{aligned}
\sup _{t \leq T}\|x(t)\|_{n} & \leq c_{0}+q_{0} \sup _{t \leq T}\|x(t)\|_{n} \int_{0}^{T}\left\|\mathrm{e}^{A(t)\left(T-t_{1}\right)}\right\|\left|t_{1}-T\right| \mathrm{d} t_{1} \\
& \leq c_{0}+q_{0} \sup _{t \leq T}\|x(t)\|_{n} \int_{0}^{T}\left\|\mathrm{e}^{A(t) u}\right\| u \mathrm{~d} u \leq c_{0}+q_{0} \hat{\chi}_{0} \sup _{t \leq T}\|x(t)\|_{n} .
\end{aligned}
$$

By (2.3), we have $q_{0} \hat{\chi}_{0}<1$. So

$$
\|x\|_{C\left(R_{+}\right)} \leq\left(1-q_{0} \hat{\chi}_{0}\right)^{-1} c_{0}=\left(1-q_{0} \hat{\chi}_{0}\right)^{-1} \nu_{A}\|f\|_{C\left(R_{+}\right)} .
$$

That is,

$$
\|\hat{U}\|_{C(0, \infty)}<\left(1-q_{0} \hat{\chi}_{0}\right)^{-1} \nu_{A} .
$$

Now condition (2.3) implies (5.16). Hence, the required results is due to Corollary 5.7.

To prove the next lemma, which is a basis of the proof of Theorem 3.1, consider the equation

$$
\dot{x}(t)=\int_{0}^{\eta} R_{1}(\mathrm{~d} \tau) x(t-\tau)
$$

where $R_{1}$ is additive with

$$
V_{1}\left(R_{1}\right):=\int_{0}^{\eta} \tau\left\|R_{1}(\mathrm{~d} \tau)\right\|_{n}<\infty
$$

Lemma 5.8. Let $Y(t)=t Z(t)$, where $Z(t)$ is the fundamental solution to (5.18). If, in addition,

$$
\|Z\|_{L^{1}}=\int_{0}^{\infty}\|Z(t)\|_{n} \mathrm{~d} t<\infty
$$

then $\|Y\|_{L^{1}} \leq\|Z\|_{L^{1}}^{2}\left(1+V_{1}\left(R_{1}\right)\right)$. 
Proof. By (5.18)

$$
\begin{aligned}
\dot{Y}(t) & =t \dot{Z}(t)+Z(t)=t \int_{0}^{\eta} R_{1}(\mathrm{~d} \tau) Z(t-\tau)+Z(t) \\
& =\int_{0}^{\eta} R_{1}(\mathrm{~d} \tau)(t-\tau) Z(t-\tau)+\int_{0}^{\eta} \tau R_{1}(\mathrm{~d} \tau) Z(t-\tau)+Z(t) .
\end{aligned}
$$

Thus,

$$
\dot{Y}(t)=\int_{0}^{\eta} R_{1}(\mathrm{~d} \tau) Y(t-\tau)+F(t)
$$

where

$$
F(t)=\int_{0}^{\eta} \tau R(\mathrm{~d} \tau) Z(t-\tau)+Z(t)
$$

Hence,

$$
Y(t)=\int_{0}^{t} Z\left(t-t_{1}\right) F\left(t_{1}\right) \mathrm{d} t_{1}
$$

By the Young inequality for convolutions, cf. [2], page 141, Theorem 9.4.1, $\|Y\|_{L^{1}} \leq\|Z\|_{L^{1}}\|F\|_{L^{1}}$. But $\|F\|_{L^{1}} \leq$ $\|Z\|_{L^{1}}\left(1+V_{1}\left(R_{1}\right)\right)$. We thus have established the required result.

Concluding remarks: We have established explicit exponential stability conditions for a class of linear multivariable retarded systems with slowly varying coefficients. As the example shows, in appropriate situations these conditions enable us to avoid the constructing of the Lyapunov type functionals.

\section{REFERENCES}

[1] B.F. Bylov, B.M. Grobman, V.V. Nemyckii and R.E. Vinograd The Theory of Lyapunov Exponents. Nauka, Moscow (1966) (in Russian).

[2] D.J. Garling, Inequalities. A Jorney into Linear Analysis. Cambridge, Cambridge Univesity Press (2007).

[3] M.I. Gil, Stability of Finite and Infinite Dimensional Systems. Kluwer, NewYork (1998).

[4] M.I. Gil, The Aizerman-Myshkis problem for functional-differential equations with causal nonlinearities. Functional Differential Equations 11 (2005) 175-185.

[5] A. Halanay, Differential Equations: Stability. Oscillation, Time Lags. Academic Press, NY (1966)

[6] J.K. Hale and S.M.V. Lunel, Introduction to Functional Differential Equations. Springer, New York (1993).

[7] N.A. Izobov, Linear systems of ordinary differential equations. Itogi Nauki i Tekhniki. Mat. Analis. 12 (1974) 71-146 (Russian).

[8] V. Kolmanovskii and A. Myshkis, Applied Theory of Functional Differential Equations. Kluwer (1999).

[9] S.G. Krein, Linear Equations in a Banach Space. Nauka, Moscow (1971) (in Russian).

[10] M. Marcus and H. Minc, A Survey of Matrix Theory and Matrix Inequalities. Allyn and Bacon, Boston (1964).

[11] J.-P. Richard, Time-delay systems: an overview of some recent advances and open problems. Automatica 39 (2003) $1667-1694$.

[12] R. Vinograd, An improved estimate in the method of freezing. Proc. Amer. Soc. 89 (1983) 125-129.

[13] A. Zevin and M. Pinsky, Delay-independent stability conditions for time-varying nonlinear uncertain systems. IEEE Trans. Automat. Contr. 51 (2006) 1482-1485.

[14] A. Zevin and M. Pinsky, Sharp bounds for Lyapunov exponents and stability conditions for uncertain systems with delays. IEEE Trans. Automat. Contr. 55 (2010) 1249-1253. 